\title{
Publisher's Note: Nonrelativistic nucleon effective masses in nuclear matter: Brueckner-Hartree-Fock model versus relativistic Hartree-Fock model \\ [Phys. Rev. C 93, 015803 (2016)]
}

\author{
A. Li ${ }^{\circledR}$, J. N. Hu, X. L. Shang, and W. Zuo
}

(Q) (Received 17 July 2020; published 24 July 2020)

DOI: 10.1103/PhysRevC.102.019907

This paper was published online on 28 January 2016 with the omission of a footnote label for the first author. Ang Li's footnote should read as "Corresponding author: liang@xmu.edu.cn". The paper has been corrected as of 20 July 2020. The paper is incorrect in the printed version of the journal. 